\title{
Gesprengte Ordnungen. Verhandlungen anarchistischer Gewalt in der französischen und spanischen Gegenwartsliteratur
}

\section{Anarchistische ,Paragesellschaften" in Geschichte und Gegenwartsliteratur}

Es braucht zwei Dinge, um eine ,Paragesellschaft‘ zu proklamieren: Rahmenbedingungen, welche die Heterogenisierung der Gesellschaft befördern, und die Wahrnehmung einer Identität, die vom institutionell legitimierten Konsens abweicht (vgl. Hiergeist 2017, 7-8). Dies lässt sich anhand der französischen und spanischen Gesellschaften des frühen 20. Jahrhunderts exemplifizieren: Die Industrialisierung bedingt dort prekäre Beschäftigungsverhältnisse für Arbeitende, während sich die Fabrikbesitzer über ihre Einkünfte meist nicht beklagen können (vgl. Vicente 2013, 111). Doch erst, als diese Arbeitenden ein Gruppenbewusstsein entwickeln, sich in sozialistischen, kommunistischen und anarchistischen Vereinigungen formieren, in deren Namen gesellschaftlich agieren und als deren VertreterInnen wahrgenommen werden (vgl. Julliard 2012, 629), kann von einer ,Paragesellschaft“ gesprochen werden. ${ }^{1}$ Denn erst in dem Moment beginnen die spannungsreichen Verhandlungen mit der ,Mehrheitsgesellschaft', die Forderungen nach Sichtbarkeit und Partizipation, die bisweilen widerständigen institutionellen Reaktionen darauf (vgl. Hiergeist 2018, 5-6); erst in dem Moment springt die Diskursmaschine an, die für die Verortung des neuen Anderen in Verhältnis zu einem bislang als einheitlich wahrgenommenen System verantwortlich ist, und sie läuft umso heißer, je mehr Schwierigkeiten beiden Seiten die Integration bereitet. Wie stark diese in ihren Positionierungen aufeinander bezogen sind und sich gegenseitig verstärken (vgl. Hiergeist 2017, 12-13), veranschaulicht abermals das Beispiel der Jahrhundertwende: Während die reaktionären Regierungen die anarchistischen Gruppierungen in Frankreich und Spanien als Gefahr für die hergebrachte Ordnung mittels Repressionen (Verboten, Sabotagen, Verhaftungen,

1 Dabei ist es meist gerade die Ausgrenzung durch eine angenommene ,Mehrheitsgesellschaft“, die zur Ausbildung des Gruppenbewusstseins führt (vgl. Hiergeist 2020, 233). 
Festnahmen, Exekutierungen) unschädlich machen wollen, ${ }^{2}$ verlangen diese wiederholt, man müsse die nationalstaatliche Souveränität und das kapitalistische Wirtschaftssystem unterminieren und eine alternative Gesellschaft ohne Herrschaft etablieren (vgl. Dugast 2001, 7), wofür sie nicht nur propagandistische Manifeste und Fiktionen verfassen (vgl. Litvak 1995, 217), Streiks organisieren (vgl. Préposiet 2002, 107) und Reformschulen und Kommunen gründen, ${ }^{3}$ sondern auch Attentate auf Machthabende und Revolutionsversuche durchführen (vgl. Lida 2008, 169; Granier 2008, 169). Solche gewaltsamen Übergriffe häufen sich vor allem ab 1904, so dass von einer „ère des attentats“ oder „oleadas del terrorismo“ die Rede ist. ${ }^{4}$

Diese anarchistischen ,Paragesellschaften“ des frühen 20. Jahrhunderts mögen auf den ersten Blick wenig mit der Gegenwartskultur zu tun haben. Nichtdestotrotz haben sie in den letzten Jahren in den französischen und spanischen Fiktionen eine verhältnismäßig große Popularität erlangt. Seit Beginn des Jahrtausends zählt man für Frankreich sechs, für Spanien zwölf Romane und Filme, die sie als Kernthema wählen. ${ }^{5}$ Der Grund für diese häufige Bezugnahme liegt sehr wahrscheinlich

2 Die Regierungen beider Länder nehmen die anarchistischen Gruppierungen als Bedrohung wahr und verwenden vor allem ab der Niederschlagung der Pariser Kommune 1871 und der Bourbonenrestauration 1875 immense Energien darauf, jegliche Bestrebungen und Aktivitäten im Keim zu ersticken. In Spanien ist die anarchistische Gewerkschaft FTRE ab 1888 untersagt, Bildungsveranstaltungen werden erschwert oder verhindert, Druckerzeugnisse systematisch beschlagnahmt und vernichtet. Wer die strengen Regeln übertritt oder auch nur eines Übertritts verdächtig wird, hat mit Verhaftungen, harten Geld- und Gefängnisstrafen oder gar mit einer öffentlichen Hinrichtung zu rechnen. Auch in Frankreich herrschen das Horrorszenario eines anarchistischen Umsturzes und ein dementsprechend repressiver Kurs und es kommt infolge der lois scélérates 1893/94 zu hunderten von Razzien, Festnahmen und Verurteilungen Maßnahmen, die sich verschärfen, je näher der Erste Weltkrieg rückt (vgl. Esenwein 1989, 59; Berry 2002, 14).

3 Bekannt geworden sind in diesem Zusammenhang etwa die Reformschulen La Ruche von Sébastien Faure in Rambouillet und die Escuela nueva von Francisco Ferrer y Guardia in Barcelona. 4 In Frankreich werden ab 1882 immer wieder Attentate verübt, vor allem in der Zeit von 1892 bis 1894 (vgl. Maitron 1975, 155 sowie 214). In Spanien häufen sich die Attentate 1893-1897 und 1904-1909 (vgl. Núnez Florencio 2010, 61-88).

5 In Frankreich erschienen die Romane Michel Ragon: Georges et Louise (2001), Hélène Zimmer: Vairon (2019), Francis Desvois: Nosotros (2019), Danielle Demangeon-Raguin: Emma. Une vie entre deux mondes (2019), Olivier Houles: Vous n'en avez pas fini avec le bonheur (2019) sowie der Spielfilm Elie Wajeman Les anarchistes (2015); in Spanien Rosa Montero: La hija del canibal (1998), Aziz Lascano: La sombra del anarquista (2006), Miquel Mir: Diario de un pistolero anarquista (2006), Montero Glez: Pólvora negra (2008), Sergio Vila-Sanjuán: Una heredera de Barcelona (2010), Jorge Navarro Pérez: Las cinco muertes del barón airado (2011), Pablo Martín Sanchez: El anarquista que se llamaba como yo (2012), Albert Villanueva: Por hacer a tu muerte compañía (2018), Agustín Comotto: El peso de las estrellas (2019) sowie die 
darin, dass sich zwischen den damaligen und heutigen gesellschaftlichen Herausforderungen Parallelen ziehen lassen. Seit im Zug der Kombination von globalisiertem Neoliberalismus und Wirtschaftskrise die Schere zwischen Arm und Reich zunehmend auseinanderklafft, hat die Zahl derer, die sich von den PolitikerInnen nicht repräsentiert fühlen, sich mit den Institutionen nicht identifizieren und sich für politische Problemlösungen jenseits der Parlamente einsetzen, in beiden Ländern nicht nur zugenommen, sondern sie haben sich untereinander auch koordiniert - man denke für Spanien etwa an die indignados-Bewegung, die 2011 und 2012 monatelang die Puerta del Sol belagert hielt, oder den movimiento okupa, der seit dem Platzen der Immobilienblase 2009 systematisch unbewohnte Gebäude besetzt, für Frankreich an die Nuit debout-Proteste gegen die Arbeitsreformen seit 2016 oder die gilets jaunes-Aktivisten in Reaktion auf die Wirtschaftspolitik seit 2018. Die Fiktionen zur anarchistischen Vergangenheit erlauben mithin auf der einen Seite die Konstruktion eines Erinnerungsorts zur Zelebration zivilgesellschaftlichen Engagements, auf der anderen aber auch die (wieder aktuelle) Verhandlung der Frage nach dem Umgang mit ,parasozialen‘ Phänomenen.

Vor allem die Terrorakte und Revolutionsversuche bilden dabei das Thema erster Wahl, schließlich ziehen sie das institutionalisierte System in radikaler Weise in Zweifel. Im Nichtanerkennen des staatlichen Machtmonopols und in der Anwendung einer Alternativexekutive ist ein expliziter Abgrenzungsgestus $\mathrm{zu}$ erkennen und sie sind als direkte Aktionen $\mathrm{zu}$ verstehen, welche die herrschaftsfreie Gesellschaftsordnung in der Tötung von wirtschaftlichen und politischen Eliten unmittelbar Realität werden lassen. Die folgenden Ausführungen widmen sich der Frage, wie die Artefakte zu den anarchistischen Gruppierungen mit deren ,Parasozialität‘ und vor allem mit der Gewalt umgehen und wie sie sie bewerten und funktionalisieren, um davon ausgehend eine Systematik unterschiedlicher Darstellungsformen von ,Paragesellschaftlichkeit' sowie ihre kulturellen Funktionen zu entwickeln. Ziel ist es, über einen Kulturvergleich Rückschlüsse auf aktuelle diskursive Konzeptualisierungen von Gesellschaftlichkeit in Frankreich und Spanien zu ziehen.

\section{Zur Fiktionalisierung anarchistischer Gewalt}

In der Ära der Attentate und in den Jahrzehnten danach wird der Anarchismus in der Literatur immer dann präferenziell thematisiert, wenn ein manichäisti-

Filme Vicente Aranda: Libertarias (1996), Marie Noelle/Peter Sehr: La mujer del anarquista (2009) und Manuel Huerga: Salvador (2006). 
scher Kampf des Staats gegen eine unberechenbare Bedrohung inszeniert werden soll (vgl. Eisenzweig 2001, 69), und die Rolle, die anarchistischen Figuren in diesem Zusammenhang am häufigsten zuteil wird, ist die der Terroristen (vgl. Vittorio 2014, 21). In seiner literatursoziologischen Studie Énigmes et complots arbeitet Luc Boltanski heraus, dass im ausgehenden 19. Jahrhundert, einem Zeitraum, der sich durch eine Intensivierung der staatlichen Kontrolle auszeichnet, mit den ,Komplottromanen“ eine eigene Gattung entstehe, die illegitime Angriffe (etwa von SozialistInnen, KommunistInnen oder AnarchistInnen) auf die etablierte gesellschaftliche Ordnung inszeniere. Ideologisches Ziel dieser Texte sei es, mittels eines Angstdiskurses die Rückbesinnung auf die nationalstaatliche Einheit zu befördern und dadurch das bestehende System zu zementieren (vgl. Boltanski 2013, 331-332). Als Beispiele hierfür können auf spanischer Seite etwa Rafael Salillas Morral el anarquista (1914) fungieren, das seinen Protagonisten als psychopathologisches Individuum depolitisiert, auf französischer Henry de Montherlants Le chaos et la nuit (1962), das die Aktivisten als faule und verblendete Spinner satirisiert. ${ }^{6}$ Passend zu den bereits angesprochenen repressiven politischen Maßnahmen, die in dem Zeitraum ergriffen werden, scheint in solchen Texten eine Gesellschaftsauffassung durch, die ,Paragesellschaftlichkeit‘ kaum zulassen kann, die Pluralität und Partikularinteressen aus Angst vor einem Kontrollverlust diskursiv opfert. Reduziert auf ihre terroristischen Intentionen inkarnieren die AnarchistInnen das Andere der Ordnung, das es in Sündenbockmanier auszumerzen gilt.

Einen grundverschiedenen Ton schlagen jene Fiktionen an, die gegenwärtig zur anarchistischen Vergangenheit erscheinen. Im Zuge des Erinnerungsbooms, der nicht nur die politischen und medialen Debatten, sondern auch die Fiktionen seit den frühen 2000ern in den beiden untersuchten Ländern entscheidend geprägt hat, ${ }^{7}$ sind zahlreiche ,Geschichten von unten' verfasst worden, welche die offizielle Version mit einer Gegendarstellung konfrontieren und dadurch relativieren. Hierüber ergibt sich ein Bruch mit der Komplottisierung, so dass die AnarchistInnen und teils auch die anarchistischen TerroristInnen zunehmend als positive Figuren rehabilitiert werden. Trotz dieser Tendenz, die diesen Narrationen gemein ist, lassen sich hinsichtlich des Umgangs mit ,Paragesellschaftlichkeit“

\footnotetext{
6 Es bildet sich folglich im sozialen Imaginären der damaligen Zeit das alterisierende Stereotyp des gefährlichen, bombenwerfenden Anarchisten heraus (vgl. Pessin 1982, 17).

7 Dieser Boom ist dem Wiederaufrollen des Ersten Weltkriegs und des Bürgerkriegs aus der Perspektive der Enkelgeneration geschuldet, das sich im Moment der Auflösung des kommunikativen Gedächtnisses manifestiert. In Spanien sind in diesem Zusammenhang zahlreiche Romane aus der Sicht der Republikaner erschienen, welche die franquistische Version parieren (vgl. SuntrupAndresen 2008, 281).
} 
zwischen den Werken französischer und spanischer Provenienz Unterschiede ausmachen, ${ }^{8}$ die im Folgenden im Vergleich von Hélène Zimmers Vairon (2019) und Michel Ragons Georges et Louise (2001) mit Alberto Villanuevas Por hacer a tu muerte compañía (2018), Agustín Comottos El peso de las estrellas (2019) und Sergio Vila-Sanjuáns Una heredera de Barcelona (2010) dargelegt werden sollen.

Vairon erzählt die Geschichte der Bauerstochter Zulma, die im ausgehenden 19. Jahrhundert in ärmlichen Verhältnissen in der französischen Provinz aufwächst und sich mit Arbeitslosigkeit und Krankheit konfrontiert nach der Geburt von Zwillingen gezwungen sieht, nach Paris abzuwandern. In der Hauptstadt kommt sie in Kontakt mit anarchistischen Gruppierungen, übernimmt nach und nach deren Ideen, gewinnt dadurch an Selbstbewusstsein, kurzum: reift vom unmündigen Bauernmädchen zur libertären Feministin. Diese Entwicklung geht mit einer Haltungsänderung einher. Während zunächst Pessimismus und Resignation vorherrschen, bewertet sie ihr Leben und ihre soziale Stellung zunehmend hoffnungsvoll:

[J]e crois en la liberté de chacun d'embrasser la voie émancipatrice qui lui sied. Ce que j'approuve c'est l'engagement pris avec soi-même, pour se libérer de la domination, en tant que principe moral.

(Zimmer 2019, 85)

Voter est notre droit. Déconstruisons ensemble les préjugés qui nous entravent.

(Zimmer 2019, 105-106)

L'oppression des femmes transcende les catégories sociales, vous le savez. Chaque femme affronte les responsabilités qui lui incombent selon les modalités imposées par son milieu culturel.

(Zimmer 2019, 133)

In diesen direkten Reden Zulmas, die in ihrer Rhetorik teils salbungsvoll, teils wie Zitate aus einem Lehrbuch für Staatsbürgerkunde wirken, verkündet Vairon die Frohbotschaft des Austritts aus der sozialen Determiniertheit mittels vernunftbasierter Selbstermächtigung. Was dabei als anarchistische Prinzipien gehandelt wird (Freiheit, Gleichheit, Wahlrecht, Selbstverantwortung), ist auffällig kongruent mit den republikanischen Grundwerten. Es geht dem Text folglich weniger um die Profilierung des Anarchismus als ,Paragesellschaft‘ denn um seine Assimilierung zum Zweck der Zementierung des Modells der repräsentativen Demokratie.

8 Dies wird bereits in quantitativer Hinsicht deutlich: Es liegen aus Spanien doppelt so viele Texte vor wie aus Frankreich. Dazu kommt, dass auch die französischen Autoren ihre Handlung nicht selten lieber im Nachbarland ansiedeln, statt in heimischen Gefilden. Der französische Anarchismus scheint sich insgesamt weniger als Erinnerungsort zu eignen als der spanische. 
Das moralisch komplexe Thema des anarchistischen Terrors wird in diesem Kontext nur tentativ angegangen. Zunächst evoziert der Text zwar wiederholt eine imminente Revolution, die innerhalb kurzer Zeit eine fundamentale Veränderung bewirken soll, jedoch wird nicht konkretisiert, mit welchen Mitteln diese umgesetzt werden soll. Die neue Gesellschaft bleibt abstrakt, behält den Status einer rhetorischen Floskel:

Dans cinq mois, il ne restera plus rien de l'idéologie immonde que vous vous fatiguez à déverser.

(Zimmer 2019, 91)

Il n’y a rien d'autre à faire que de désirer le chaos. Désirer le chaos uniquement. Rompre les associations d'idées. Les associations tout court. L'union se fait de toute façon. Si l'on veut se rapprocher d'une réalité profonde, il faut anénatir le sens. L'univers est un chantier sans contremaître, Zulma, tu as raison. Le désordre est la vérité. ～(Zimmer 2019, 128)

Ist die Verhandlung von Gewalt aus Gründen der Geschichtstreue unumgänglich, so werden vorwiegend Szenen gezeigt, in denen die AnarchistInnen als Opfer der repressiven Polizeigewalt erscheinen. Wenn Polizisten etwa Streiks mittels Festnahmen und Erschießungen beendet, dann erscheint dies als Missachtung demokratischer Grundrechte, ${ }^{9}$ die Übergriffe der AktivistInnen sind als Reaktionen auf eine präzedent ausgeübte institutionelle Gewalt legitimiert. Selbst die Schilderung einer Straßenschlacht zwischen Ordnungskräften und Revolutionären, die sich vor dem Zug zuträgt, in dem Zulma sitzt, banalisiert die Fragwürdigkeit von Gewalt:

Dehors, un violent désir de justice secoue le train. L'homme à côté de Zulma hausse un sourcil dès que le wagon se soulève, sans refermer son journal. Une femme allongée dans l'allée centrale ronfle tranquillement, une bouteille entre les bras. Une série de tirs arrête tout, un instant; la rage du dehors et la peur du dedans. Des chevaux musclés traversent la foule. Les uniformes fracassent ce qui dépasse. La foule s'unit encore. Se presse à nouveau sur le train. Crache sur les carreaux. Renverse le train. Les passagers se brisent les uns sur les autres. Dehors, encore des tirs. Les corps s'étendent sur les voies. Se ruent sur les escaliers. Remontent dans la rue. Jettent des casseroles, des sabots. „Assassins! C'est la fin!“ Les balles se croisent. Les fusils pointent vers le cœurs enragés. Les revolvers visent les casques inflexibles.

(Zimmer 2019, 154-155)

Trotz der auf den ersten Blick externen Fokalisierung - es werden in kurzen, asyndetisch gereihten Sätzen singuläre Aktionen registriert - findet eine Hierarchisierung der Gewalt statt: Euphemistische Formulierungen wie „un violent désir de justice“ oder „la rage“ camouflieren und autorisieren die Übergriffe der Umstürzler.

9 Dies wird an den tendenziösen Formulierungen ersichtlich, der Anarchismus sei ein „mouvement immédiatement réprimé par Clemenceau qui ne reconnaît pas le droit de grève aux fonctionnaires“ (Zimmer 2019, 68). 
Auch die Tatsache, dass die Gewehre der Polizei auf die Herzen, die Revolver der AnarchistInnen hingegen auf die Helme zielen, lässt erstere brutaler erscheinen als zweitere. Nicht zuletzt ist die Drastik der Situation durch die Erwähnung von im sicheren Kokon des Zugs schlafenden und zeitungslesenden Figuren und die unpersönliche Darstellung der Revolutionäre reduziert. Die Szene ist in ihrer Eindringlichkeit folglich dezidiert gedämpft, bleibt etwa hinter der detailreichen, blutigen und hochemotionalen Beschreibung der Geburt von Zulmas Babys zu Beginn des Romans weit zurück. Über diskursive Strategien der Tabuisierung, Trivialisierung und Distanzierung verschleiert Vairon die anarchistische Gewalt und stellt die Frage nach ihrer Legitimität der Feier der ,mehrheitsgesellschaftlichen' Werte nach.

Auf ähnliche Strategien rekurriert Michel Ragons Georges et Louise, welches die Lebensgeschichte von Louise Michel erzählt, ihren Kampf für die Pariser Kommune, ihr reformpädagogisches Engagement, ihre Aktivität in der Arbeiterbewegung, ihre Gefangenschaft in der neukaledonischen Strafkolonie, ihr Exil in London und ihre Rückkehr nach Frankreich. Bereits die im Titel anklingende Grundidee des Romans, ihre Biografie in der intellektuellen und emotionalen Verflechtung mit dem damaligen Ministerpräsidenten Georges Clemenceau darzustellen, unterstreicht die Intention der Republikanisierung der Anarchistin. Gezeichnet wird sie ausschließlich positiv, wenn nicht gar heroisierend in ihrem Einsatz für die Rechte der Arbeitnehmer und für eine unparteiische Justiz. Wenn sie Brot an die Armen verteilt, marginalisierten Frauen zur Emanzipation verhilft, aufgrund ungerechter Gesetze ${ }^{10}$ unschuldig inhaftiert und verurteilt wird, ${ }^{11}$ bündelt sie als Inkarnation der Gleichheit und Brüderlichkeit/Schwesterlichkeit die Sympathien der repräsentativdemokratischen Lesenden. Dies kulminiert in ihrer Beerdigung am Ende des Texts: Die 2000 Gäste, die anwesenden Politiker und die Polizeieskorte inszenieren die Anarchistin nicht als Angehörige einer staatszersetzenden Splittergruppe, sondern verorten sie als Nationalheldin im Zentrum der Gesellschaft. ${ }^{12}$

10 Es heißt, die lois scélérates seien „lois pour remprimer l'agitation anarchiste et syndicale“ (Ragon 2001, 156) oder „le congrès parlementaire de Londres m'auraitfait anarchiste“ (Ragon 2001, 176).

11 Louise Michel wird, laut Roman, unschuldig angeklagt, weil sie den Diebstahl von Brot in einer Bäckerei befürwortet haben soll und kommt dafür nach nicht ganz objektiven Ermittlungen und einem tendenziös geführten Prozess für fünf Jahre ins Gefängnis (Ragon 2001, 100).

12 Flankiert wird diese Darstellung dadurch, dass wiederholt betont wird, SozialistInnen und AnarchistInnen seien in dieser Zeit so unterschiedlich nicht gewesen, hätten gleiche Ziele verfolgt und unter den gleichen Repressionen gelitten (Ragon 2001, 129, 176). 
In der Darstellung anarchistischer Gewalt verfährt Georges et Louise banalisierend. Die Erschießungen von Reaktionären, die sie während der Pariser Kommune vornimmt, sind zu robin-hoodesken Akten der Barmherzigkeit stilisiert: „[P]our empêcher qu’on tuât, elle tuait“ (Ragon 2001, 27), heißt es; außerdem sei sie jedem, den sie angeschossen habe, unmittelbar zu Hilfe geeilt, um ihn medizinisch zu versorgen (Ragon 2001, 27). Über ihre Sympathie für die Attentäter merkt der Text relativierend an: „Elle exalte les attentats anarchistes en France, en même temps elle avoue beaucoup aimer en Angleterre la reine Victoria“ (Ragon 2001, 162), so dass ihre extremistische Haltung willkürlich und mithin wenig ernstzunehmend erscheint. ${ }^{13}$ So zentral ist hier die erinnerungsörtliche Funktion, das Zementieren des Einheitsgedankens, dass es die ,paragesellschaftliche‘ Komponente verdrängt.

Auf den ersten Blick verhält es sich in den untersuchten spanischen Texten ähnlich, sie stimmen mit den französischen in der positiven, nicht-stigmatisierenden Darstellung der anarchistischen Protagonisten überein: In Por hacer a tu muerte compañía etwa wird keiner der geplanten Anschläge realisiert, die Figuren brechen die Ausführung häufig ab oder werden an ihr gehindert. Sehr wohl ausführlich erzählt und rhetorisch aufgeladen sind hingegen die Repressionen der Exekutive, ${ }^{14}$ so dass die AnarchistInnen verhältnismäßig friedfertig als Opfer eines Systems erscheinen, das sie ungerechtfertigterweise alterisiert und verfolgt. ${ }^{15}$ El peso de las estrellas, das den Anarchismus entdogmatisierend und einer Vergruppung entgegenwirkend weniger als „una secta, una ideología“ sehen möchte denn als „actitud existencial“ (Comotto 2019, 220), ergreift Partei für die Anschläge, indem es sie als Reaktion auf eine jahrelange Unterdrückung, als Notwehr gegen ein illegitimes Unrecht von oben konstruiert. ${ }^{16}$ In Una heredera de Barcelona entwickeln Lesende mit der Hauptfigur, einem eingangs konservativ gezeichneten Anwalt, der den Fall eines unschuldig des

13 Überhaupt scheint Louise Michel weniger als Handelnde denn als statische Verkörperung republikanischer Werte, zumal ihr Portrait eher berichtend denn erzählend gestaltet ist.

14 Als Beispiel kann die durch die kurzen, anaphorischen Sätze rhetorisierte Passage dienen, welche die Erschießung eines Anarchisten dramatisiert: „Pocos minutos antes de las seis de la tarde, Francesc Layret recibe la primera de las balas en la cara. La bala que le duele. La bala que hace que aquel andamiaje de hierro y acero deje de sostenerlo. La bala que hace que no preciba todas las otras que entran en su cuerpo. La bala que hace que note cómo su sangre se le escapa del cuerpo para crear charcos viscosos en la acera. La bala que lo separa para siempre de sus ideas, de sus anhelos, de su vida“ (Villanueva 2018, 76).

15 Ausgestellt wird die Perspektive des Regierungspräsidenten, der die GewerkschafterInnen als „plaga y el gran veneno que estaba emponzonando la convivencia“ bezeichnet und nach dem „antídoto“ der repressiven Verfolgung greift (Villanueva 2018, 59).

16 Es ist die Rede von einer „gran violencia desatada por parte de pistoleros a sueldo de la patronal, que dejó muchos muertos en el camino, nueva política represiva“ (Comotto 2019, 20). 
Terrorismus bezichtigten Jungen übernimmt, nach und nach Sensibilität für die Belange der ,niederen' Schichten. Während dieser der anarchistischen Gewalt zu Beginn des Romans noch mit Entsetzen begegnet, ${ }^{17}$ versteht er sie zunehmend als Antwort auf das Gebaren der Obrigkeit ${ }^{18}$ und als ehrenwerten (obschon fruchtlosen) Versuch, das Kippen Spaniens in eine Diktatur zu verhindern. Die anarchistischen Gruppierungen erscheinen in diesen Texten folglich als Teil Spaniens, denen nach jahrzehntelanger stigmatisierender Dominanz faschistischer Geschichtsversionen endlich eine Stimme verliehen wird. Wie in den französischen Romanen wird die Gewalt zugunsten der Propagierung einender republikanischer Werte und unter dem rhetorischen Deckmantel des Pluralismus, der - klassisch repräsentativdemokratisch - als Einheit der Vielheit, als gerade durch das Heterogene Homogene konzeptualisiert ist, ausgeklammert.

Nichtsdestotrotz verhalten sich die spanischen Texte in der Modellierung dieser Botschaft weniger monolithisch als die französischen. Dies ist erstens der Verdopplung der zeitlichen Ebenen geschuldet, die sie vornehmen. In Por hacer a tu muerte compañia alterniert der temporale Fokus kapitelweise zwischen den 1920er Jahren und der Gegenwart, in El peso de las estrellas besorgt ein Dialog zweier Zeitgenossen über die anarchistische Frühphase und die heutige Politik ein zeitliches Oszillieren und in Una heredera de Barcelona leitet der ausführliche Prolog des (fiktiven?) Herausgebers aus dem Jahr 2010 die Narration aus dem Vorkriegsspanien ein. Dies lässt die Vergangenheit weniger als Realität denn als Konstruktion ex post erscheinen und sorgt für Polyphonie, so dass die Parteilichkeit und Inauthentizität von Geschichtsdarstellungen implizit mitthematisiert werden, ihre evaluativen Positionierungen weniger festgeschrieben und mithin revidierbar wirken.

Mit dieser Verdopplung der Zeitebenen einher geht, dass die Texte allesamt Beobachter zweiter Ordnung integrieren, denn sie weisen nicht nur Vermittlungsinstanzen auf, die den anarchistischen ProtagonistInnen folgen, sondern auch Figuren, welche die Konstruktion dieser Narration bewerkstelligen oder überwachen. In Por hacer a tu muerte compañía lässt sich eine Geschichtsdozentin der Universität Barcelona in einem Provinzarchiv anstellen, um mittels Quellenstudium und Zeitzeugenbefragung die Geschichte ihres Großvaters zu rekonstruieren; in El peso

17 In einem Gespräch mit einer Anarchistin klagt er diese an: „siguen matando inocentes, amenazando industriales y desafiando la legislación; y que manipulan al pueblo puro, el buen pueblo que busca prosperar sin nihilismos ni tergiversaciones“ (Vila-Sanjuán 2010, 37).

18 Es heißt: „Las bombas a la autoridad: lo hacen cuando radicalizan una desconfinanza y un resentimiento que sin duda son inustos y equivocados, pero precisamente por ello deberíamos ser los primeros interesados en paliarlos. Los que tenemos fortuna, los que nacimos privilegiados, estamos más obligados a fomentar la paz social que los que han venido al mundo sin nada y loh an tenido después todo en contra“ (Vila-Sanjuán 2010, 255). 
de las estrellas stößt ein Intellektueller bei seinen Bibliotheksrecherchen auf einen ehemaligen Aktivisten, mit dem er sich verabredet, um gemeinsam seine Biographie zu verfassen; in Una heredera de Barcelona entdeckt ein Journalist auf dem Speicher die Aufzeichnungen seines verstorbenen Großvaters und fühlt sich zu deren Veröffentlichung verpflichtet. ${ }^{19}$ Diese Figuren besorgen nicht nur eine gewisse Präferenz des Analytischen vor dem Erleben, des telling vor dem showing, ${ }^{20}$ sondern auch einen hohen metanarrativen Anteil und mise-en-abyme-Strukturen, da man als LeserIn eben das Buch in Händen hält, das die jeweiligen impliziten AutorInnen abfassen. Die Techniken betonen die Vermitteltheit der Erzählung und schaffen eine Distanz zwischen Lesendem und Vergangenheit, die eine Reflexion über die Verortung der anarchistischen Gruppierungen innerhalb der Gesellschaft anstoßen kann.

Hierzu passend charakterisieren sich die untersuchten Romane über die explizite oder implizite Problematisierung der Wahrheit von Geschichte. Por hacer a tu muerte compañía präsentiert die Historie nicht als Akkumulation objektiver Fakten, sondern als Vehikel persönlicher Sinnstiftung. Die Protagonistin Julia diagnostiziert bei sich und ihrer Familie eingangs eine Identitätsstörung, zu deren Auflösung die bislang tabuisierte anarchistische Biographie des Großvaters den Schlüssel bildet. ${ }^{21}$ Auch El peso de las estrellas streicht die Unmöglichkeit einer verlässlichen Rekonstruktion der Vergangenheit heraus, indem es die Fallibilität der Erinnerung akzentuiert. ${ }^{22}$ Una heredera de Barcelona thematisiert die Schwierigkeit der Differenzierung zwischen Fakt und Fiktion, indem es die Erzählung als Hybride zwischen Tagebuch und Roman, also gleichsam als Autofiktion des Großvaters, präsentiert. ${ }^{23}$ Dies verweist darauf, dass die Bewer-

19 Genau genommen ist in Una heredera de Barcelona noch eine weitere Beobachterebene eingefügt dadurch, dass die Hauptfigur als Journalist auch wieder Beobachtungen der Gegenwartsgesellschaft vornimmt.

20 Es ergibt sich aufgrund der Rahmenhandlung der Geschichtsrecherche bzw. der professionellen Interviewsituation ein dokumentarischer Gestus angesichts der dargestellten Vergangenheit insofern als zahlreiche, mit Jahreszahlen versehene Fakten präsentiert werden.

21 Julia proklamiert hier eingangs: „estoy segura de que dentro de mí ya no hay órganos, ni vísceras. Soy solo una carcasa exterior. Una piel que envuelve una nebulosa obsesión“ (Villanueva 2018, 9) - eine Entfremdung, die sich im Laufe des Romans durch die Ergründung der Geschichte auflöst, so dass sie abschließend in einer familiären, freundschaftlichen und partnerschaftlichen Heimat ankommt.

22 Es heißt: „todos tenemos mucha dificultad para recordar los sucesos de nuestra infancia“ (Comotto 2019, 8), schließlich stünde die Identitätsstiftung und nicht die Archivierung von Fakten im Vordergrund.

23 Auch explizit wird im Prolog gefragt: „qué hay de realidad y qué hay de ficción en estas páginas“ (Vila-Sanjuán 2010, 14). 
tung der anarchistischen Gruppierungen in den Texten eine konstruierte ist, die an subjektive Interessen und Machtansprüchen gekoppelt ist.

Überdies lässt sich für die Texte eine eher schwache Position der ErzählerInnen konstatieren. Diese treten nicht als Autoritäten mit doktrinärem Wahrheitsanspruch auf, sondern als homodiegetische Subjekte. Por hacer a tu muerte compañia präsentiert dem Lesenden nicht die abschließenden Ergebnisse der Recherchen der Historikerin Julia, sondern er entdeckt zusammen mit ihr wie in einer Detektivgeschichte schrittweise neue Informationen, so dass die Rekonstruktion des Vergangenen als work in progress erscheint. In El peso de las estrellas tritt die Vermittlungsinstanz hinter den hohen dialogischen Anteilen, die das Gespräch mit dem Anarchisten über seine Vergangenheit impliziert, passagenweise völlig zurück, so dass die geäußerten Bewertungen unmissverständlich als subjektiv und als Momentaufnahmen eines unabschließbaren Aushandlungsprozesses markiert sind. Una heredera de Barcelona konfrontiert den Rezipienten mit dem Tagebuch, das als Egodokument seine perspektivische Gebundenheit ausstellt und zur kompensatorischen Imagination von Gegenversionen einlädt.

Die Funktion der genannten Elemente liegt meines Erachtens nicht allein in der Relativierung einer zu Zwecken der Identitätsstiftung unkomplex und kantenlos gestalteten Vergangenheit, vielmehr ikonisieren sie auch eine Subversivität, die sich am inhaltlichen Dogmatismus reibt. Die Inszenierungsstrategien fungieren als Subtext, der eine Abweichung vom ,mehrheitsgesellschaftlichen' Gestus transportiert, schließlich wird mit der Ausräumung autoritärer, hierarchischer und doktrinärer Strukturen aus der Narration sowie mit seiner Auffassung von der Untrennbarkeit von Fakt und Fiktion, von Kunst und Leben - ein dezidiert anarchistischer Gestus bedient. ${ }^{24}$ Am deutlichsten wird dieses Kokettieren mit anarchistischen Prinzipien im Anspruch, die reale Gesellschaft durch die Texte zu verändern. Dementsprechend betont El peso de las estrellas wiederholt, soziales Unrecht sei eine universelle Konstante, die es unter Primo de Rivera und Franco genauso zu bekämpfen gelte wie unter Chirac und Sánchez, und endet mit einem offenen Brief an den amtierenden spanischen Präsidenten, der die Annullierung der 2007 verabschiedeten Ley de la Memoria Histórica fordert. ${ }^{25}$ Auch den anderen

24 Die Ästhetik zahlreicher anarchistischer Texte zeichnet sich durch die Transgression bestehender und als Beschränkung wahrgenommener Konventionen, da Postulat konkreter politischer, sozialer und ethischer Nützlichkeit, sowie die nachdrückliche Betonung des performativen Charakters von Literatur und Kunst, ihr Verständnis als militante und revolutionäre Akte, aus (vgl. Coquio 1999, 487; Torres Planells 2006, 351).

25 Dieses Gesetz, das eine Anerkennung der Kriegsopfer beider Seiten, eine Verurteilung des Franco-Regimes und die Exhumierung von Opfern franquistischer Unterdrückung fordert, 
beiden Romanen eignet diese pragmatische Dimension, proklamieren sie doch die Schaffung einer alternativen Realität, indem sie einer von institutioneller Seite jahrzehntelang unterdrückten „Wahrheit“ Raum verschaffen. ${ }^{26}$ Durch dieses Verständnis von Literatur als direkter Aktion werden die dargestellten anarchistischen Gruppierungen als ,Paragesellschaften“ in ihrer dialogischen Bezogenheit auf und in ihrer Abweichung vom Konstrukt ,Mehrheitsgesellschaft‘ sichtbar, so dass die Texte über eine komplexitätsreduzierende Instrumentalisierung des Vergangenen für die Zementierung des gegenwärtigen Systems hinausgehen.

\section{Fazit}

Aus diesen Analysen lassen sich mehrere Schlüsse ziehen. Erstens: Das Verschwinden von Komplottnarrativen zu anarchistischen Gruppierungen zeigt, dass deren diskursive Alterisierung nicht mehr zeitgemäß ist, möglicherweise, weil die Distanz eine größere Toleranz gegenüber der Abweichung zulässt, möglicherweise aber auch, weil ein so radikaler Exklusionsgestus mit dem Ethos des Pluralismus unvereinbar ist, der anders als der frühe Nationalstaatsgedanke vertritt, dass soziale Einheit nicht aus faktischer Homogenität, sondern aus dem Zusammenschluss des Heterogenen resultiert. Zweitens: Es besteht ein inszenatorischer Unterschied zwischen den französischen Werken, die anarchistische Gruppierungen ausschließlich in Übereinstimmung mit republikanischen Werten zeichnen, sie mithin assimilieren, und den spanischen, welche ihnen zusätzlich einen ,paragesellschaftlichen“ Charakter zugestehen, die Verflochtenheit mit und Abweichung von der ,Mehrheitsgesellschaft‘ zur Anschauung bringen, sie folglich integrieren. Ein Grund für diese Differenz könnte sein, dass der Stellenwert der Idee der sozialen Homogenität in Frankreich aufgrund der zentralistischen Tradition sinnstiftender ist als in Spanien, wo seit Jahrhunderten eine eher regionalistische Prägung vorherrscht. Es könnte auch sein, dass in Frankreich das Interesse an der Zelebrierung des kollek-

reicht dem Roman nicht weit genug, da die Urteile, die durch franquistische Gerichte verhängt wurden, nicht annulliert wurden, so dass den Opfern der Repressionen nicht ausreichend Gerechtigkeit widerfahren ist (Comotto 2019, 373).

26 Por hacer a tu muerte compañía konstruiert eine ausgeprägte Mystery-Spannung um die Biographie des Großvaters, indem sie diese als hochgradig relevantes, jedoch kaum lösbares Geheimnis konstruiert. Die wiederholten Versuche der Protagonistin Julia, die Wahrheit aufzudecken, zerschellen nicht selten an einer Mauer des Schweigens, so dass auch die Motivation der Lesenden zur Ergründung der Geschichte steigen mag. Una heredera de Barcelona verleiht durch den ausführlichen Prolog dem Akt der Publikation als Wiedergutmachung eines Versäumnisses eine besondere Bedeutung. 
tiven Gedächtnisses und damit verbunden der Wunsch nach der Zementierung des bestehenden Systems größer ist als am Ausloten der Möglichkeiten sozialen Wandels durch ,Paragesellschaften“, dass also gegenwärtig eine etwas konservativere Grundstimmung herrscht, zumal innerhalb der Gattung des Romans, die dort seit dem Realismus dezidiert eine identitätsstiftende Funktion übernimmt. Ein Grund könnte auch sein, dass die etwas andere Konnotierung terroristischer Gewalt in beiden Ländern für den Unterschied verantwortlich ist, schließlich haben in Frankreich die Präsidenten und Medien die BürgerInnen infolge islamistischer Anschläge in den vergangenen Jahren wiederholt als Opfer einer externen Bedrohung inszeniert, während in Spanien die terroristische Gewalt eventuell aufgrund der Erfahrungen mit der ETA und anderen radikalen SeparatistInnen - etwas stärker als Internum wahrgenommen wird, das aus der Gesellschaft heraus geschieht.

\section{Bibliographie}

Berry, David. A History of the French Anarchist Movement, 1917-1945. Westport: Greenwood, 2002.

Biersack, Martin, Teresa Hiergeist und Benjamin Loy. „Das Leben der Anderen. Historische, soziologische und narrative Dimensionen paralleler Sozialität“. Parallelgesellschaften. Instrumentalisierungen und Inszenierungen in Politik, Kultur und Literatur (Romanische Studien, Beihefte 8). Hg. Martin Biersack, Teresa Hiergeist und Benjamin Loy. München: AVM, 2019. 5-17.

Boltanski, Luc. Rätsel und Komplotte. Kriminalliteratur, Komplotte, moderne Gesellschaft. Frankfurt a.M.: Suhrkamp, 2013.

Comotto, Agustín. El peso de las estrellas. Barcelona: Rayo Verde, 2019.

Coquio, Catherine. „Le soir et l'aube. Décadence et anarchisme“. Revue d'histoire littéraire de la France 99.3 (1999): 453-466.

Eisenzweig, Uri. Fictions de l'anarchisme. Paris: Bourgois, 2001.

Esenwein, Georges Richard. Anarchist Ideology and the Working-Class Movement in Spain, 1868-1898. Berkeley: University of California Press, 1989.

Dugast, Jacques. La vie culturelle en Europe au tournant des XIXe et XX siècles. Paris: PUF, 2001.

Frigerio, Vittorio. La littérature de l'anarchisme. Anarchistes de lettres et lettrés face à l'anarchisme. Grenoble: ELLUG, 2014.

Granier, Caroline. Les briseurs de formules. Les écrivains anarchistes en France à la fin du XIXe siècle. Coeuvres: Ressouvenances, 2008.

Hiergeist, Teresa. „Selbst, anders, neu. Reflexionen zu den kulturellen und ästhetischen Bedeutungen von ,Parallel- und Alternativgesellschaften'“. Parallel- und Alternativgesellschaften in den Gegenwartsliteraturen. Hg. Teresa Hiergeist. Würzburg: Königshausen \& Neumann, 2017. 7-24. 
Hiergeist, Teresa. „Explosive Utopien. Gesellschaftliche Einheit und anarchistischer Terrorismus im sozialen Imaginären der Jahrhundertwende“. Imaginationen des Sozialen. Narrative Verhandlungen zwischen Integration und Divergenz (1750-1945). Hg. Benjamin Loy, Simona Oberto und Paul Strohmaier. Heidelberg: Winter, 2020. 221-236.

Julliard, Jacques. Les gauches françaises, 1762-2012. Histoire, politique et imaginaire. Paris: Flammarion, 2012.

Lida, Clara E. „La Primera Internacional en España, entre la organización pública y la clandestinidad (1868-1889)“. Tierra y libertad. Cien años de anarquismo en España. Hg. Julián Casanova. Barcelona: Crítica, 2010. 33-60.

Litvak, Lily. „La prensa anarquista 1880-1913“. El anarquismo español y sus tradiciones culturales. Hg. Bert Hofmann, Pere Joan i Tous und Manfred Tietz. Frankfurt a.M.: Vervuert, 1995. 201-244.

Maitron, Jean. Le mouvement anarchiste en France (Bd. 1). Des origines à 1914. Paris: Gallimard, 1975.

de Montherlant, Henry. Le chaos et la nuit. Paris: Éditions Lidis, 1962.

Núnez Florencio, Rafael. „El terrorismo“. Tierra y libertad. Cien años de anarquismo en España. Hg. Julián Casanova. Barcelona: Crítica, 2010. 61-88.

Pessin, Alain. La rêverie anarchiste. 1848-1914. Paris: Librairie des méridiens, 1982. Préposiet, Jean. Histoire de l'anarchisme. Paris: Tallandier, 2002.

Ragon, Michel. Georges et Louise. Paris: Albin Michel, 2001.

Salillas, Rafael. Morral el anarquista. Madrid: Librería de los Sucesores de Hernando, 1914. Suntrup-Andresen, Elisabeth. Hacer Memoria. Der Bürgerkrieg in der Literatur der Nachgeborenen. München: Meidenbauer, 2008.

Torres Planells, Sonya: „Art i anarquia“. La rosa il-lustrada. Trobada sobre cultura anarquista $i$ lliure pensament. Hg. Associació Cultural Alzina i Clemente Penalva Sant Vicent del Raspeig. Alicante: Publicacions de la Universitat d'Alacant, 2006. 133-148.

Vicente, Laura. Historia del anarquismo en España. Madrid: Catarata, 2013.

Vila-Sanjuán, Sergio. Una heredera de Barcelona. Barcelona: Ediciones Destino, 2010. Villanueva, Alberto. Por hacer a tu muerte compañía. Almería: Editorial Círculo Rojo, 2018. Zimmer, Hélène. Vairon. Paris: P.O.L., 2019. 